\title{
Stem Cell Therapy
}

\author{
Eyman Elsayed Mohammed, Osama Bakr Sadeek, Hasnaa Ahmed Abo-Elwafa Abd \\ Elhady Ragab Abd El-Gawad,Medhat Ibrahim and Amany Abbass \\ Department of Clinical and Chemical Pathology, Faculty of Medicine, Sohag \\ University
}

\begin{abstract}
Introduction: Stem cells have an interesting history, it traces from mid of 1800 to the date, and it remains the most controversial issue of research even today. The ability of stem cells to self-renew and give rise to generations with variable degrees of differentiation capacities, offers significant potential for generation of tissues that can replace diseased and damaged ones.

Aim of the work: Aim of this essay to clarify different types and sources of stem cells and their potential uses to treat many diseases.

Conclusion: The potency of the stem cell specifies the differentiation potential and is an important factor that is responsible for the characteristic nature of the stem cells because of this tissue renewing capacity, stem cell researches are now one of the important cornerstones for medical research and development.
\end{abstract}

Keywords: Stem cells. Therapy.

\section{Introduction}

Stem cells (SC) have an interesting history, it traces from mid of 1800 to the date, and it remains the most controversial issue of research even today ${ }^{(\mathbf{1})}$.

The ability of SC to self-renew and give rise to siblings with different degrees of differentiation capabilities, offers important chance for generation of tissues to replace diseased or damaged tissues in the body, with no or minimal side effects, complications or risk of rejection ${ }^{(2)}$.

Stem cell therapy is an amazing medical advancement that goes straight to the source of the problem and treats different disorders like muscular dystrophy, Alzheimer's disease, atherosclerosis, diabetes, spinal cord injury, Parkinson's disease, Rheumatoid Arthritis, leukemia and many others ${ }^{(3)}$.

\section{Aim of the work:}

Aim of this essay was to clarify different types and sources of stem cells and their potential uses to treat many diseases.

\section{What are stem cells?}

There are three basic categories of cells that make-up the human body: germ cells, somatic cells and SC. Germ cells are cells that give rise to gametes, i.e. eggs and sperm while somatic cells include the bulk of the cells making-up the human adult and each of these cells in its differentiated state has its own copy, or copies of the genome; the only exception being cells without nuclei, i.e. red blood cells ${ }^{(4,5)}$.

SC are cells found in nearly all body organisms that can divide and differentiate into differentiated cells and can self-renew to produce more SC. They have the extraordinary potential to develop into many other different cell types in the body during early life and growth. In many tissues SC serve as a sort of internal repair system and dividing essentially without limit to replenish other cells ${ }^{(1)}$.

SC can be divided into two types, non-definitive and definitive SC. Non-definitive SC have the capacity of developing into any organ or tissue of the body. The best example is the 
fertilized egg, but the most commonly known are embryonic SC (ESC) and induced pluripotent stem (iPS) cells. Definitive SC are derived from nondefinitive $\mathrm{SC}$ and are organ or tissue specific. They are responsible for maintaining the organ or tissue ${ }^{(6)}$.

Definitive SC can be divided into two types; those that maintain continuously proliferating cell systems and those that maintain partially proliferating cell systems ${ }^{(7,8)}$.

\section{Stem cell therapy}

\section{Stem cells with hematological diseases:}

SC are the key ingredient of hematopoietic cell grafts. They have been used clinically since 1968 when the first successful bone marrow transplant (BMT) was performed at the University of Minnesota. Hematopoietic cell transplantation (HCT) has become a well-established treatment modality for malignant and nonmalignant diseases. Hematopoietic SC (HSCs), the best-described SC, are categorized as multipotent $\mathrm{SC}$ because they self-renew, differentiate into all the cells of the blood lineage, and reconstitute the hematolymphoid system for the life of an individual ${ }^{(9)}$.

Whether sourced from bone marrow, umbilical cord blood or, predominantly today from mobilized peripheral blood, the hematopoietic stem cell has developed into the most common source of cells for cell-based therapies for both hematological and non-hematological diseases ${ }^{(10)}$.

\section{Stem cell treatment for non- malignant blood disorders:-}

Allogenic BMT/HSCT is a curable treatment for groups of non malignant blood disorders if an adequate donor is available. The first group of disorders includes sickle cell anemia, thalassemia, Fanconi anemia and other genetic diseases affecting blood and immune cells (both of which are generated from HSCs). The target of these transplantations is to replace the patient's defective HSCs with normal HSCs from an allogenic donor. The second group of blood and immune diseases, such as aplastic anemia or other forms of autoimmune diseases, the patient's HSCs either become defective later in life or destroyed by other aberrant cells. Allogenic BMT/HSCT Will help to replace or rebuild the pool of functional HSCs ${ }^{(11)}$.

In sickle cell disease (SCD), The goal of SCT is removal of the sickle RBCs along with its cellular progenitors and replacement with donor hematopoietic pluripotent SC which give rise to normal RBCs expressing no sickle hemoglobin (HbS). This will lead to reduce $\mathrm{HbS}$ levels to those associated with the trait condition. It can prevent many serious complications from SCD which can cause widespread morbidity and early mortality ${ }^{(\mathbf{1 2})}$.

Myeloablative allogeneic hematopoietic stem-cell transplantation (HSCT) is the possible curative option for patients with SCD or thalassemia major. Successful allogeneic SCT not only eliminates the sickle-cell-induced vaso-occlusive symptomatology, but also can induce reversal of some end organ damage which might had occurred before SCT ${ }^{(13)}$. Moreover, the new advances in stem cell gene therapy may provide a safer therapy for SCD in the near future ${ }^{(14,15)}$.

\section{Stem Cell Treatment for blood cancers}

Over the past half century, One of the most commonly used stem cell applications was the transplantation of healthy bone marrow into a patient who was suffering from a disease that destroys the body's immune system such as leukemia ${ }^{(16)}$.

Leukemia and other diseases like it result in an immunodeficiency disorder caused by improperly 
functioning bone marrow. Leukemia is a type of cancer that is commonly associated with children and young adults destroying the bone marrow, which is the tissue that contains HSCs. These cells are responsible for making all the cellular components of blood such as white blood cells, red blood cells, and platelets. Leukemia results in the body having a high level of immature white blood cells, which in turn can lead to the likelihood of the patient becoming increasingly susceptible to infections and weakness as the illness progresses ${ }^{(16)}$.

One way is to throw out all the abnormal WBCS, thus allowing healthy WBCs to grow in their place; this can be done by chemotherapy ${ }^{(16)}$. The usual doses of chemotherapy drugs may induce many significant and even dangerous side effects to all quickly dividing tissues such as the bone marrow or gonads. Higher doses of these drugs might be more effective, but they are not given because they could severely damage the bone marrow. This could lead to lifethreatening infections, bleeding, and other problems due to low blood cell counts ${ }^{(17)}$

The other way is to carry out the bone marrow transplant. To do this, all patient's existing bone marrow cells and abnormal WBCs are first killed using a combination of chemotherapy and radiotherapy. Then, donor bone marrow containing healthy $\mathrm{SC}$ is introduced into the patient's blood. If the transplantation is successful, the $\mathrm{SC}$ will migrate into the patient's bone marrow and begin producing healthy WBCs to replace abnormal ones $(\mathbf{1 7}, \mathbf{1 8})$.

\section{Complications of hematopoietic stem cell transplantation}

The risk and the type of complications depend on the preparative regimen received prior to HSCT, the age of the patient at time of HSCT, the presence of comorbid conditions, and the time between the treatment and follow-up ${ }^{(16)}$.

\section{Stem cells with Cardiovascular diseases (CVDs):}

Cardiovascular disease is a leading cause of death worldwide and becomes increasingly prevalent in the elderly population. Irrespective of the etiology, most cardiovascular diseases eventually lead to heart failure (HF), which is progressive and irreversible. Furthermore, despite modern advances in therapy and management, the number of annual deaths due to CVDs worldwide continues to increase; by 2030 , it is expected that nearly 23.6 million people will die from heart diseases including $\mathrm{HF}^{\text {(19-21) }}$.

In the last decade, a variety of stem cell types, including skeletal myoblasts, bone marrow-derived SC (e.g., bone marrow mononuclear cells), circulating progenitor cells and mesenchymal SC, have been utilized in the treatment of patients with acute myocardial infarction(AMI) or chronic ischemic cardiomyopathy in clinical trials ${ }^{(22,23)}$.

\section{Stem cells with diabetes mellitus:}

Diabetes mellitus is a metabolic disorder characterized by hyperglycemia due to reduced insulin production or insulin resistance in the body. Specifically in type 1 diabetes insulin production is markedly reduced due to the destruction of insulin producing $b$ cells as a result of autoimmunity. On the other hand, type 2 diabetes results from a combined effect of insulin resistance and reduced insulin secretion by b cells. While type 2 diabetes can be sometimes managed through the use of oral hypoglycemic agents or lifestyle changes, type 1 patients are exclusively dependent on insulin injections for their whole life $\operatorname{span}^{(24)}$.

In the progression of type 2 diabetes, $\beta$ cells undergo many 
complex changes and $\beta$ cell mass declines gradually: $\beta$ cells first compensate to hyperglycemia by secreting more insulin; once fail, $\beta$ cells become dysfunction with the marked impairment of insulin secretion and phenotypic changes; finally, $\beta$ cells become apoptotic. Current data suggested that $72 \%$ recipients of islets transplantation became insulinindependent ${ }^{\text {(24) }}$.

To overcome the shortage of available pancreas or islets for transplantation, several types of SC have been used to make pancreatic $\beta$ cells, including human embryonic SC (hESCs)/induced pluripotent SC (iPSCs), pancreatic stem/progenitor cells, and non-pancreatic SC. There is also evidence of adult $\beta$ cells regeneration through $\beta$ cell replication and cellular reprogramming ${ }^{(25)}$.

\section{Stem cells with ocular diseases:}

Cornea: LESC deficiency is pathological, either partially or completely, caused by mechanical injury, chemical exposure, burns or may be developed by some diseases such as Stevens Johnson syndrome. Treatment of such conditions may be done by SCT using LESC transplantation therapy ${ }^{(\mathbf{2 6})}$.

Lens: Lens predecessor cells have been derived from human ESCs as well as iPSCs. Lens SC may have a role in maintaining the lens transparency and might be important in the pathogenesis of cataract or other lens abnormalities ${ }^{(27)}$.

Retina: Most therapeutic studies have been done on animal retinal disease models. Diseases in the inner retina include ischemic retinopathy and optic neuropathy, which cause irreversible damage in the retinal ganglion cells and amacrine cells ${ }^{\text {(28) }}$.

\section{Stem Cells: Ethical Issues}

Stem cell technology is speedily increasing within the field of regenerative medicine, granting $\mathrm{DE}$ novo production of functional tissue and providing for brand new diagnostic and therapeutic capabilities that will surpass the risk benefit ratio of typical existing reparative treatment modalities e.g. organ transplantation, rejuvenation of tissues ${ }^{(29)}$.

The use of human embryonic tissues for research has a moral problem as it brings two highly valued but conflicting moral principles: the inherent duty to provide treatment to ease pain and suffering on one hand and the value of human life and dignity on the other. Extraction of SC from human embryos violates the second principle as it leads to destruction of potential human life. Both principles cannot coexist together, but which principle takes precedence is a rather contentious issue. How the embryo should be considered from moral or legal point of view is the main debatable issue associated with Human Embryonic SC research ${ }^{(30)}$.

The moral status that the human embryo is given varies. Three different main positions with variations can be separated:

1- Having full moral status after fertilization of the egg.

2- Having a moral status that begins with deserving protection and increases as the fertilized egg becomes more human-like.

3-Having no moral status at all, regarded as organic material, with a status no different from other body parts ${ }^{(30)}$.

\section{Conclusion:}

Cells are long-lived cells in the body that have the ability to differentiate into a specialized cell to create new tissues. The potency of the stem cell specifies the differentiation potential and is an important factor that is responsible for the characteristic nature of the stem cells because of this tissue renewing capacity, stem cell researches are now one of the 
important cornerstones for medical research and development.

\section{References:}

1. Cruz DM, Gomes M, Reis RL, Moratal D, Salmeron-Sanchez M, Ribelles JL, et al. Differentiation of mesenchymal stem cells in chitosan scaffolds with double micro and macroporosity. $\mathbf{J}$ Biomed Mater Res A. 2010;95(4):1182-93.

2. Gupta K, Chandran S, Hardingham GE. Human stem cell-derived astrocytes and their application to studying Nrf2-mediated neuroprotective pathways and therapeutics in neurodegeneration. $\mathrm{Br} \mathrm{J}$ Clin Pharmacol. 2013;75(4):907-18.

3. Villa C, Erratico S, Razini P, Farini A, Meregalli M, Belicchi M, et al. In vivo tracking of stem cell by nanotechnologies: future prospects for mouse to human translation. Tissue Eng Part B Rev. 2011;17(1):1-11.

4. Jeffery R, Poulsom R, Alison MR. Sources of adult hepatic stem cells: haematopoietic. Methods Mol Biol. 2009;481:141-54.

5. Alison MR, Islam $\mathrm{S}$. Attributes of adult stem cells. J Pathol. 2009;217(2):144-60.

6. Blanpain $\mathrm{C}$, Simons $\mathrm{BD}$. Unravelling stem cell dynamics by lineage tracing. Nat Rev Mol Cell Biol. 2013;14(8):489-502.

7. Gancz D, Gilboa L. Insulin and Target of rapamycin signaling orchestrate the development of ovarian niche-stem cell units in Drosophila. Development. 2013;140(20):4145-54.

8. Gancz D, Gilboa L. Hormonal control of stem cell systems. Annu Rev Cell Dev Biol. 2013;29:13762.

9. Copeland N, Harris D, Gaballa MA. Human umbilical cord blood stem cells, myocardial infarction and stroke. Clin Med (Lond). 2009;9(4):342-5.

10. Malgieri A, Kantzari E, Patrizi MP, Gambardella S. Bone marrow and umbilical cord blood human mesenchymal stem cells: state of the art. Int J Clin Exp Med. 2010;3(4):248-69.

11. Brodsky RA, Chen AR, Dorr D, Fuchs EJ, Huff CA, Luznik L, et al. High-dose cyclophosphamide for severe aplastic anemia: long-term follow-up. Blood. 2010;115(11): 2136-41.

12. Khoury R, Abboud MR. Stem-cell transplantation in children and adults with sickle cell disease: an update. Expert Rev Hematol. 2011;4(3):343-51.

13. Krishnamurti L, Kharbanda S, Biernacki MA, Zhang W, Baker KS, Wagner JE, et al. Stable longterm donor engraftment following reduced-intensity hematopoietic cell transplantation for sickle cell disease. Biol Blood Marrow Transplant. 2008;14(11):1270-8.

14. Hsieh MM, Kang EM, Fitzhugh $\mathrm{CD}$, Link MB, Bolan CD, Kurlander R, et al. Allogeneic hematopoietic stem-cell transplantation for sickle cell disease. N Engl J Med. 2009;361(24):2309-17.

15. Hsieh MM, Fitzhugh CD, Tisdale $\mathrm{JF}$. Incidence of second cancers after allogeneic hematopoietic stem cell transplantation using reduceddose radiation. Blood. 2009;114(1): 225; author reply -6 .

16. Tian H, Qu Q, Liu L, Wu D. Advances in Stem Cell Therapy for Leukemia. Curr Stem Cell Res Ther. 2016;11(2):158-65.

17. Burnett J, Newman B, Sun D. Targeting cancer stem cells with natural products. Curr Drug Targets. 2012;13(8):1054-64.

18. Rane SR, Kulkarni MM, Puranik SC. Chronic neutrophilic leukemia: 
a rare case report. Indian J Hematol Blood Transfus. 2014;30(Suppl 1):77-9.

19. Izarra A, Moscoso I, Canon S, Carreiro C, Fondevila D, MartinCaballero $\mathrm{J}$, et al. miRNA-1 and miRNA-133a are involved in early commitment of pluripotent stem cells and demonstrate antagonistic roles in the regulation of cardiac differentiation. J Tissue Eng Regen Med. 2017;11(3):787-99.

20. Peran M, Marchal JA, Lopez E, Jimenez-Navarro M, Boulaiz $\mathrm{H}$, Rodriguez-Serrano F, et al. Human cardiac tissue induces transdifferentiation of adult stem cells towards cardiomyocytes. Cytotherapy. 2010;12(3):332-7.

21. Armbruster $\mathrm{C}$, Tevaearai $\mathrm{H}$, Kleber A, Carrel T, Giraud M. Strategies to induce cardiac differentiation of Mesenchymal Stem Cells. J Stem Cells Regen Med. 2007;2(1):210-1.

22. Hansson EM, Lindsay ME, Chien KR. Regeneration next: toward heart stem cell therapeutics. Cell Stem Cell. 2009;5(4):364-77.

23. Hansson M, Olesen DR, Peterslund JM, Engberg N, Kahn M, Winzi M, et al. A late requirement for Wnt and FGF signaling during activininduced formation of foregut endoderm from mouse embryonic stem cells. Dev Biol. 2009;330(2): 286-304.

24. Halban PA, German MS, Kahn SE, Weir GC. Current status of islet cell replacement and regeneration therapy. J Clin Endocrinol Metab. 2010;95(3):1034-43.

25. $\mathrm{Wu}$ SM, Hochedlinger $\mathrm{K}$. Harnessing the potential of induced pluripotent stem cells for regenerative medicine. Nat Cell Biol. 2011;13(5):497-505.

26. Dhamodaran K, Subramani M, Ponnalagu M, Shetty R, Das D. Ocular stem cells: a status update! Stem Cell Res Ther. 2014;5(2):56.

27. Qiu X, Yang J, Liu T, Jiang Y, Le $\mathrm{Q}, \mathrm{Lu} \mathrm{Y}$. Efficient generation of lens progenitor cells from cataract patient-specific induced pluripotent stem cells. PLoS One. 2012;7(3):e32612.

28. Laouri M, Chen E, Looman M, Gallagher M. The burden of disease of retinal vein occlusion: review of the literature. Eye (Lond). 2011;25(8):981-8.

29. Zacharias DG, Nelson TJ, Mueller PS, Hook CC. The science and ethics of induced pluripotency: what will become of embryonic stem cells? Mayo Clin Proc. 2011;86(7):634-40.

30. Karakaya A, Ilkilic I. Ethical Assessment of Human Embryonic Stem Cell Research According to Turkish Muslim Scholars: First Critical Analysis and Some Reflections. Stem Cell Rev. 2016;12(4):385-93. 